\title{
Erratum to: A modified two-factor multivariate analysis of variance: asymptotics and small sample approximations
}

\author{
Solomon W. Harrar • Arne C. Bathke
}

Published online: 7 June 2012

(C) The Institute of Statistical Mathematics, Tokyo 2012

\section{Erratum to: Ann Inst Stat Math (2012) 64:135-165 DOI 10.1007/s10463-010-0299-0}

The authors regret the following errors which, unfortunately, were not caught during the write-up, revision and typesetting.

Line 4 on page 148 should read

$$
f=\frac{\operatorname{tr}\left(\sum_{i=1}^{n} c_{i i} \Sigma_{i}\right)^{2}+\left[\operatorname{tr}\left(\sum_{i=1}^{n} c_{i i} \Sigma_{i}\right)\right]^{2}}{\sum_{i=1}^{n} \sum_{j=1}^{n} c_{i j}^{2}\left[\operatorname{tr}\left(\Sigma_{i} \Sigma_{j}\right)+\operatorname{tr}\left(\Sigma_{i}\right) \operatorname{tr}\left(\Sigma_{j}\right)\right]} \quad \text { and } \quad \Psi=\frac{1}{f} \sum_{i=1}^{n} c_{i i} \Sigma_{i} .
$$

Also in the formulae for $f_{\psi}$ for $\psi \in\{A, A|B, B, B| A, A B, G\}$ on pages 148 and 149; $\operatorname{tr}\left(\Sigma^{2}\right)$ should be replaced with $\operatorname{tr}\left(\Sigma^{2}\right)+(\operatorname{tr} \Sigma)^{2}, \operatorname{tr}\left(\Sigma_{i j}^{2}\right)$ should be replaced with $\operatorname{tr}\left(\Sigma_{i j}^{2}\right)+\left(\operatorname{tr} \Sigma_{i j}\right)^{2}, \operatorname{tr}\left(\Sigma_{i j} \Sigma_{i j^{\prime}}\right)$ should be replaced with $\operatorname{tr}\left(\Sigma_{i j} \Sigma_{i j^{\prime}}\right)+\operatorname{tr}\left(\Sigma_{i j}\right) \operatorname{tr}\left(\Sigma_{i j^{\prime}}\right)$, $\operatorname{tr}\left(\Sigma_{i j} \Sigma_{i^{\prime} j^{\prime}}\right)$ should be replaced with $\operatorname{tr}\left(\Sigma_{i j} \Sigma_{i^{\prime} j^{\prime}}\right)+\operatorname{tr}\left(\Sigma_{i j}\right) \operatorname{tr}\left(\Sigma_{i^{\prime} j^{\prime}}\right)$ and $\operatorname{tr}\left(\Sigma_{i j} \Sigma_{i^{\prime} j}\right)$ should be replaced with $\operatorname{tr}\left(\Sigma_{i j} \Sigma_{i^{\prime} j}\right)+\operatorname{tr}\left(\Sigma_{i j}\right) \operatorname{tr}\left(\Sigma_{i^{\prime} j}\right)$.

The address of second author has meanwhile changed to "305C Multidisciplinary Science Building, Lexington, KY 40536-0082, USA".

The online version of the original article can be found under doi:10.1007/s10463-010-0299-0.

S. W. Harrar $(\varangle)$

Department of Mathematical Sciences, The University of Montana,

32 Campus Drive, Missoula, MT 59812, USA

e-mail: harrar@mso.umt.edu

\section{A. C. Bathke}

Department of Statistics, University of Kentucky,

305C Multidisciplinary Science Building, Lexington, KY 40536-0082, USA

e-mail: arne@uky.edu 